\title{
Growth and detection of filamentous fungi in the BacT/Alert blood culture system
}

\author{
A Breathnach, J Evans
}

\begin{abstract}
Little is known about the behaviour of filamentous fungi in most blood culture systems, despite their increasingly recognised role in infections of immunocompromised hosts. The ability of the BacT/Alert system (Organon Teknika, Durham, North Carolina, USA) to detect the growth of 19 such fungi was examined. Eleven species grew and were detected rapidly; two species did not grow. Six species grew slowly, and were generally only recovered with terminal subculture after prolonged incubation. The $\mathrm{CO}_{2}$ production graph for some of these fungi showed a slow but steady rise, insufficient to cause the apparatus to signal positive. These results show that the BacT/Alert system may miss some fungi, either because of no growth in the medium or undetected slow growth. The latter problem could be overcome by prolonged incubation and terminal subculture when fungal infection is considered likely. Alteration of the signalling mechanism might permit earlier detection of some slow growing fungi.

(f Clin Pathol 1995;48:670-672)
\end{abstract}

Keywords: BacT/Alert, blood cultures, filamentous fungi.

Filamentous fungi are an increasingly important cause of infection in the immunocompromised host, accounting for much morbidity and mortality in these patients. The diagnosis of systemic fungal infection still presents many difficulties and is frequently established only by histological examination of biopsy or necropsy samples. ${ }^{1}$ Biopsies are invasive and in many patients the presenting features are vague, with little indication of which organs to biopsy. Serological tests often lack both sensitivity and specificity, each test only detects one species of fungus and they are rarely available outside reference centres. ${ }^{2}$ Molecular biological techniques are similarily not widely available. Blood cultures are simple and safe, are widely used and occasionally yield a diagnosis of fungal infection. However, relatively little is known about the behaviour of non-yeast (mycelial) fungi in most blood culture systems. Failure to diagnose fungi by this method may be due to either an inability of the blood culture system to detect the fungus, or the absence of fungal spores or hyphae from the bloodstream. Most blood culture systems are designed primarily to detect the growth of bacteria and yeasts, and in trials of these systems mycelial fungi have seldom been isolated. ${ }^{3}$ This is the case even with systems designed to optimise the isolation of fungi. ${ }^{4}$ The BacT/ Alert system (Organon Teknika, Durham, North Carolina, USA) ${ }^{5}$ is a recently introduced automated blood culture system which can rapidly isolate many bacteria and yeasts. ${ }^{6}$ We examined its ability to support and detect the growth of a range of pathogenic filamentous fungi.

\section{Methods}

The BacT/Alert blood culture system is an automated system in which the base of each blood culture bottle contains an indicator which responds to a change in $\mathrm{CO}_{2}$ concentration by a change in reflectance: the incubator contains a photometric apparatus which measures this reflectance. Repeated measurements are analysed electronically and the apparatus signals positive when there is either a sustained rise in reflectance of a certain minimum rate, an acceleration in the rate of reflectance increase or a high initial reflectance. There are three types of bottle: aerobic, anaerobic and paediatric. The adult aerobic and anaerobic media are based on triptic soy broth, and the paediatric bottles contain a smaller volume of medium based on brain heart infusion broth with a reduced concentration of liquid, the intention being to allow better growth of fastidious organisms.

Nineteen strains of potentially pathogenic fungi obtained from a mycology reference laboratory (table) were inoculated into paediatric and adult aerobic blood culture bottles. Anaerobic bottles were not used because the fungi examined would not be expected to grow anaerobically. The inoculum was between 10 and $130 \mathrm{cfu}$; this was obtained by making serial dilutions of a suspension of the fungus in distilled water and subculturing fixed volumes onto Sabouraud's dextrose agar (Oxoid) to establish the colony count. Whole human blood was also added to each bottle $(5 \mathrm{ml}$ for adult bottles, $1 \mathrm{ml}$ for paediatric bottles), and the 
Results for 19 species of fungi cultured in the BacT/Alert blood culture system

(1) Growth rapidly detected by apparatus in both bottles Aspergillus flavus Aspergillus fumigatus Aspergillus nidulans Aspergillus niger* Aspergillus tereus

(2) No growth detected by apparatus (fungus isolated from one or both bottles after prolonged incubation)
Exophiala dermatidis*
Penicillium sp. Phialophora richardsio

(3) Growth detected in paediatric bottle only

(fungus isolated from aerobic bottle after prolonged incubation)

4) No growth

Acremonium sp.

Fusarium moniliformi

Fusarium oxysporum

Paecilomyces varioti

Scedosporium apiospermum

Visible fungal growth inside bottle.

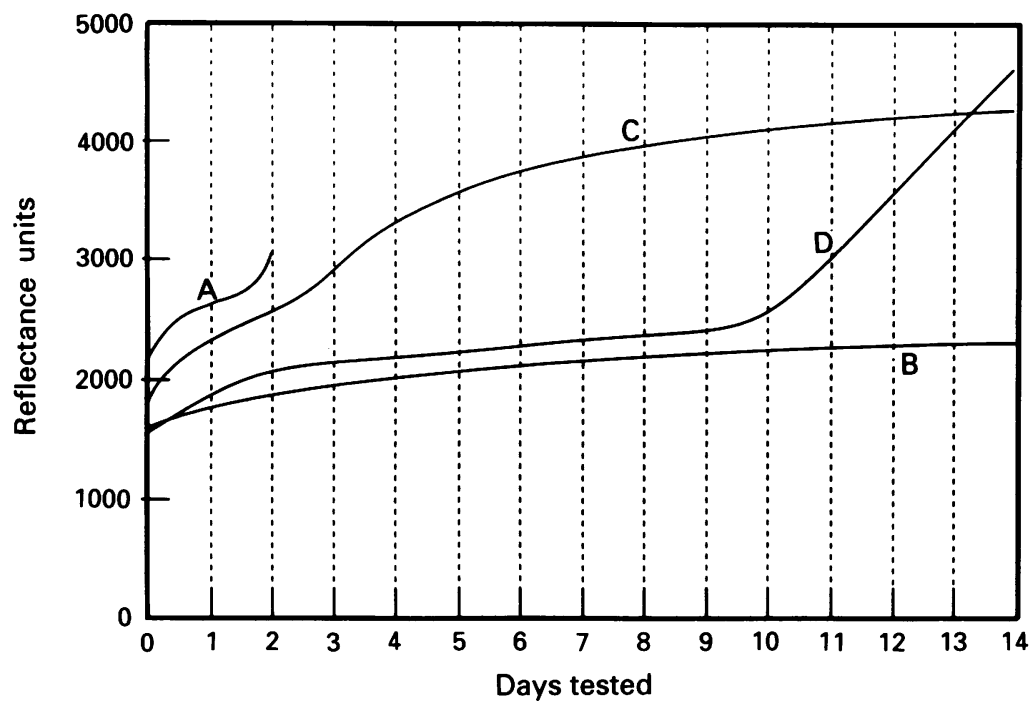

Reflectance curves (indicating $\mathrm{CO}_{2}$ production) for selected fungi. A: Fusarium moniliformis: signal positive at two days. B: Acremonium sp.: no growth at any stage. C: Exophiala spinifera: growth on terminal subculture, not detected by apparatus.

D: Phialophora richardsiae: growth on terminal subculture, not detected by apparatus.

bottles were then vented. An aliquot was taken from each bottle at this stage and cultured as above to establish a baseline colony count. The bottles were loaded into the incubator and incubated for 14 days at $35^{\circ} \mathrm{C}$, unless they signalled positive before this. When the bottles were removed, they were examined for obvious fungal growth, and subcultured onto Sabouraud's agar and blood agar (Oxoid). Following incubation of these plates for up to five days in air at $30^{\circ} \mathrm{C}$, colony counts were noted and the identity of the colonies confirmed. ${ }^{7}$ Those bottles which remained negative at 14 days were kept at room temperature and examined and subcultured as above at 21-35 days. A graph of the repeated measurements of reflectance, indicating metabolic activity, was obtained for each bottle.

\section{Results}

As shown in the table, 11 of the 19 fungi examined grew well and were quickly detected in both paediatric and aerobic bottles (the mean time to detection was 1.9 days). Only two of the fungi examined (Acremonium sp. and Alternaria alternata) failed to grow in either bottle, even with prolonged incubation. Of the remaining six species, two (Bipolaris hawaiiensis and Exophiala spinifera) were detected in the paediatric bottle after three to five days of incubation; they did not signal positive in the aerobic bottle, but were recovered after prolonged incubation. Four species grew slowly, either in both bottles ( $E$ dermatitidis) or in the paediatric bottle only (Penicillium sp., Phialophora richardsiae and $P$ verrucosa). These did not signal positive, and were recovered by subculture at 14 to 35 days.

Several of the bottles examined at the end of incubation, or after one to two weeks at room temperature, had visible growth of fungus within. In two of these cases ( $B$ hawaiiensis, Aspergillus niger) repeated subculture yielded very few colonies, no more than the baseline colony count.

When the graphs of the serial reflectance readings for each bottle were examined, we noted that, among those bottles which failed to signal positive, some of those which subsequently yielded colonies gave readings which rose steadily, compared with genuinely negative bottles which gave a virtually flat trace (figure).

\section{Discussion}

This study shows that, while the majority of the fungi examined were easily detected by the BacT/Alert system, a significant number grew slowly and escaped detection, and some species did not grow at all. It is important to note that the inocula we used, while small, may still be much larger than that of a clinical specimen. While many of these fungi are obviously capable of growth at $35^{\circ} \mathrm{C}$ ( $A$ fumigatus can grow at up to $50^{\circ} \mathrm{C}$ ), some environmental fungi may be outside their optimum growth range at this temperature, accounting for the poor growth of some strains tested. The observation that two species of fungi gave visible growth inside the bottle despite the lack of increase in colony counts on subculture may be due to the fact that in each of these cases the fungal mass did not appear particularily friable: $B$ hawaiiensis was adherent to the glass, and $A$ niger formed a dense floating mycelium, and it is possible that they failed to release spores or viable hyphae into the broth.

These findings confirm the general limitations of blood cultures in isolating fungi. The results also show that an automated system may fail to detect organisms with a low rate of metabolism (figure). A modification of the algorithm to detect slow but sustained rises in $\mathrm{CO}_{2}$ production might overcome this problem.

The yield of fungi was increased by a factor of $27 \%$ in this study, and by similar factors in other studies, ${ }^{8}$ by the practice of prolonged incubation and terminal subcultures. We doubt that this would be worthwhile in routine laboratory practice for several reasons: it would entail considerable effort and expense, the absolute yield would be small, and the information gained, while interesting, would often be too late to be of relevance. However, clinicians and microbiologists should consider this option when fungal infection is suspected, particularily as the tendency with the BacT/ Alert system seems to be to shorten the duration of incubation. ${ }^{9}$ 
We are grateful to Organon Teknika UK Ltd for supplying the blood culture bottles for this study, to Dr Colin Campbell, Bristol Public Health Laboratory and Mycology Reference Laboratory, for supplying the fungal isolates used in the study, and to Dr M Sprott, Newcastle Public Health Laboratory, for assisting in the preparation of the manuscript.

1 Musial CE, Cockerill FR III, Roberts GD. Fungal infection of the immunocompromised host: clinical and laboratory of the immunocompromised host: clinical

2 Hopwood V, Evans EGV. Serological tests in the diagnosis and prognosis of fungal infections in the compromised and prognosis of fungal infections in the compromised
patient. In: Warnock DW, Richardson MD, eds. Fungal infection in the compromised patient. 2nd edn. Chichester John Wiley and Sons, 1991:311-53.

3 Weinstein MP, Mirrett S, Wilson ML, Harrel LJ, Stratton CW, Reller LB. Controlled evaluation of BACTEC Plus26 and Roche Septi-Chek aerobic blood culture bottles. $\mathcal{F ~ C l i n}$ Microbiol 1991;29:879-82.

4 Bille J, Stockman L, Roberts GD, Horstmeier CD, Ilstrup DM. Evaluation of a lysis-centrifugation system for recovery of yeasts and filamentous fungi from blood. $\mathcal{F}$ Clin Microbiol 1983;18:469-71.

5 Thorpe TC, Wilson ML, Turner JE, DiGuiseppi JL, Willert M, Mirrett S, et al. BacT/Alert: an automated colorimetric microbial detection system. F Clin Microbiol 1990;28:160812.

6 Wilson ML, Weinstein MP, Reimer LG, Mirrett S, Reller LB. Controlled comparison of the BacT/Alert and BACTEC $660 / 730$ nonradiometric blood culture systems. $\mathcal{F}$ Clin Microbiol 1992;30:323-9.

7 Larone DH. Medically important fungi: a guide to identification 2nd edn. Washington DC: American Society for Microbiology, 1993.

8 Weinstein MP, Mirrett S, Reimer LG, Reller LB. Effect of agitation and terminal subcultures on yield and speed of the Oxoid Signal blood culture system versus the BACTEC radiometric system. $\mathcal{F}$ Clin Microbiol 1989;27:427-30.

9 Wilson ML, Mirrett S, Reller LB, Weinstein MP, Reimer LG. Recovery of clinically important microorganisms from the BacT/Alert blood culture system does not require testing for seven days. Diagn Microbiol Infect Dis 1993;16:31-4.

\title{
Longstanding proliferation of $\mathrm{CD} 3$ negative large granular lymphocytes preceding the development of high grade non-Hodgkin's lymphoma
}

\author{
S R Smith, P G Middleton, P J Birch, L Morgan, P W G Saunders
}

\section{Department of Haematology, Newcastle General Hospital, Westgate Road, Newcastle upon Tyne NE4 6BE \\ S R Smith \\ P W G Saunders \\ Department of Pathology P J Birch}

Regional Immunology Laboratory L Morgan

LRF Remission Unit Medical Molecular Biology Group, University of Newcastle upon Tyne P G Middleton

Correspondence to: Dr S R Smith.

Accepted for publication 13 October 1994

\begin{abstract}
A patient with a persistent CD3 negative large granular lymphocyte (LGL) proliferation with immunophenotypic and functional characteristics of natural killer cells is described. The LGL proliferation persisted and six years after diagnosis the patient developed a high grade $B$ cell nonHodgkin's lymphoma. Molecular studies demonstrated clonal $B$ cell populations in the peripheral blood, distinct from that identified in the lymphoma, both at presentation with non-Hodgkin's lymphoma and at complete remission following combination chemotherapy. It is postulated that $T$ cell dysregulation associated with the CD3 negative LGL proliferation may have led to $B$ cell dysfunction and loss of normal $B$ cell control, with the subsequent development of a clonal $B$ cell lymphoproliferative disorder.

(f Clin Pathol 1995;48:672-675)
\end{abstract}

Keywords: CD3 negative large granular lymphocytes, non-Hodgkin's lymphoma.

Large granular lymphocyte (LGL) proliferations are being increasingly recognised. ${ }^{1}$ New classifications have been proposed to describe LGL proliferations based on immuno- phenotypic profiles, replacing the numerous and sometimes confusing terms that have previously been used. ${ }^{23}$ High grade lymphoproliferative disorders developing in association with LGL proliferations have been reported, and include aggressive natural killer (NK) cell leukaemia, and lymphomas of large granular lymphocytes. ${ }^{4-6} \mathrm{CD} 3+$ LGL proliferations have been associated with low grade non-Hodgkin's lymphoma, ${ }^{7}$ however to our knowledge, a chronic CD3 - NK type LGL proliferation preceding the development of high grade $B$ cell non-Hodgkin's lymphoma has not been described. We report the clinical, histological, immunophenotypic, functional, and molecular characteristics of a chronic CD3 - LGL proliferation that preceded the development of a high grade non-Hodgkin's lymphoma by six years and discuss the possible causal link between the LGL lymphocytosis and $B$ cell tumour.

\section{Case report}

A 43 year old white man was noted to have a lymphocytosis when admitted for a left inguinal hernia repair in February 1987. A full blood count showed a white cell count (WBC) of $16.4 \times 10^{9} / 1$ with $84 \%$ lymphocytes, the majority of which were typical LGLs, and $12 \%$ neutrophils. Haemoglobin was $15.3 \mathrm{~g} / \mathrm{dl}$ and 